10,18

\title{
Бозонный пик в аморфном графене в рамках модели устойчивых случайных матриц
}

\author{
() И.О. Райков ${ }^{1}$, Д.А. Конюх ${ }^{2}$, А.Н. Ипатов ${ }^{3,1}$, Д.А. Паршин ${ }^{3,1, \uparrow}$ \\ ${ }^{1}$ Санкт-Петербургский академический университет РАН, \\ Санкт-Петербург, Россия \\ ${ }^{2}$ Физико-технический институт им. А.Ф. Иофффе РАН, \\ Санкт-Петербург, Россия \\ ${ }^{3}$ Санкт-Петербургский политехнический университет, \\ Санкт-Петербург, Россия \\ E-mail: conyuh.dmitrij@yandex.ru
}

Поступила в Редакцию 11 июня 2020 г.

В окончательной редакции 11 июня 2020 г.

Принята к публикации 24 июня 2020 г.

\begin{abstract}
Исследовано влияние беспорядка в распределении атомных силовых констант на оптические и акустические фононы в скалярной модели кристаллического графена как для колебаний лежащих в плоскости листа, так и для изгибных мод. Показано, что при достаточной степени беспорядка, в рамках модели устойчивых случайных матриц, обладающих трансляционной симметрией, возникает избыточная, по сравнению с дебаевской, плотность колебательных состояний бозонный пик. С ростом амплитуды относительных флуктуаций силовых констант и уменьшением среднего модуля Юнга системы, бозонный пик смещается в сторону более низких частот. При относительно слабом беспорядке (или в отсутствие беспорядка), в плотности состояний $g(\omega)$ имеется два пика, которые соответствуют логарифмическим сингулярностям ван-Хова для акустических и оптических фононов кристаллического графена. По мере увеличения беспорядка эти пики уширяются и сливаются в один, бозонный пик. Причем первыми за счет беспорядка разрушаются оптические фононы, а акустические фононы плавно трансформируются в бозонный пик. Для изгибных мод картина немного другая. По-прежнему беспорядок размывает сингулярности ван-Хова, но приводит к появлению в системе фононов, которые формируют бозонный пик и движутся вместе с ним в сторону малых частот при возрастании степени беспорядка.
\end{abstract}

Ключевые слова: аморфный графен, бозонный пик, случайные матрицы.

DOI: $10.21883 /$ FTT.2020.11.50069.126

\section{1. Введение}

В настоящее время одним из важных разделов современной теории конденсированных сред является изучение такого интересного материала, как аморфный графен. Он, как и кристаллический графен, представляет собой моноатомный плоский слой атомов углерода, в котором каждый атом связан валентной $s p^{2}$-связью с тремя своими ближайшими соседями в двумерной решетке [1-4] (см. рис. 1). Как известно, кристаллический графен, благодаря уникальным особенностям его зонной структуры, обладает исключительными механическими $[5,6]$, физическими $[7,8]$ и оптическими свойствами [9], которые находят применения [10] в различных областях. Отметим здесь его рекордно большую теплопроводность [11].

Аморфный графен исследован далеко не так подробно как кристаллический. Его электрические свойства резко отличаются от таковых у его кристаллического аналога $[2,3,12]$. Также было замечено, что аморфный графен имеет высокие прочностные качества по сравнению со своей кристаллической модификацией [13-15]. Теплопроводность аморфного графена была изучена теорети- чески $[16,17]$ в рамках модели $[18,19]$, где было впервые введено такое важное понятие как дифбузон.

В силу имеющегося в системе беспорядка, упругие силы между атомами аморфного графена флуктуируют. В связи с этим, большой интерес представляет изучение колебательных свойств аморфного графена в зависимости от амплитуды этих флуктуаций. Известно,
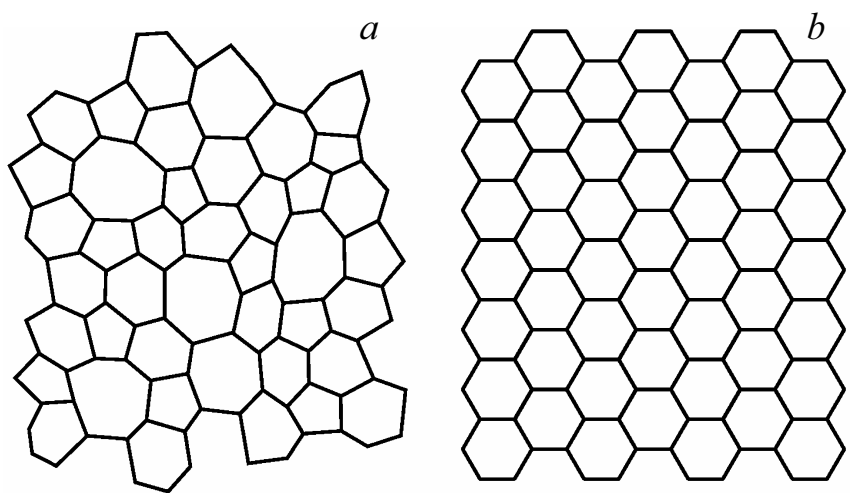

Рис. 1. Монокристаллический графен $(a)$ и аморфный графен $(b)$. 
что практически все аморфные тела в низкочастотной области колебательного спектра обладают общими универсальными свойствами. Одним из таких свойств является бозонный пик, характеризующий избыточную, по сравнению с дебаевской, плотность колебательных состояний $g(\omega) / g_{D}(\omega)$. Несмотря на большое число работ, посвященных бозонному пику, его общепринятая физическая интерпретация до сих пор отсутствует [20-25].

Некоторые авторы связывают появление бозонного пика с сингулярностью ван-Хова поперечных акустических фононов в соответствующих кристаллах с одним атомом в элементарной ячейке [26,27]. Однако в решетках с более чем одним атомом в элементарной ячейке, наряду с акустическими фононами, существуют и оптические фононы. Простейшим примером такого материала и является монослой графена. В нем есть продольные и поперечные акустические и оптические фононы, лежащие в плоскости графена, и две сингулярности ван-Хова. В этом случае связь бозонного пика с сингулярностями ван-Хова требует отдельных исследований, и изучение бозонного пика в аморфном графене представляет собой особый интерес.

Также следует принимать во внимание, что кроме планарных колебательных мод в монослое графена имеется еще один тип колебаний, перпендикулярных его плоскости - так называемые изгибные моды. Особенность этих колебаний заключается в том, что при малых частотах они, как предполагается по аналогии с теорией упругости [28], имеют закон дисперсии $\omega \propto q^{2}$ и соответственно очень малую скорость распространения при малых импульсах $\hbar q$. Однако вопрос о влиянии беспорядка на изгибные моды до сих пор остается открытым. В силу симметрии, изгибные моды никак не взаимодействуют с планарными модами графена (фононами). В связи с этим, возникает интерес рассмотреть изгибные колебания аморфного графена на предмет наличия в них бозонного пика. Ожидается, что при сильном беспорядке в системе изгибные моды исчезнут c появлением бозонного пика, который тогда будет иметь чисто фононное происхождение. Одной из целей нашей работы является проверить это предположение.

Настоящая работа имеет следующую структуру. В разд. 2 описываются имеющиеся в кристаллическом графене типы колебаний, а также обосновывается наш выбор скалярной модели. В разд. 3 представлен вывод закона дисперсии фононов, поляризованных в плоскости листа графена, а в разд. 4 получена их плотность колебательных состояний в рамках скалярной модели. В разд. 5 описываются изгибные моды кристаллического графена с квадратичным законом дисперсии. В разд. 6 представлен подход для описания колебаний аморфного графена, основанный на использовании теории случайных матриц. Наконец, в разд. 7 приводятся полученные плотности колебательных состояний и находятся важные закономерности, связанные с бозонным пиком.

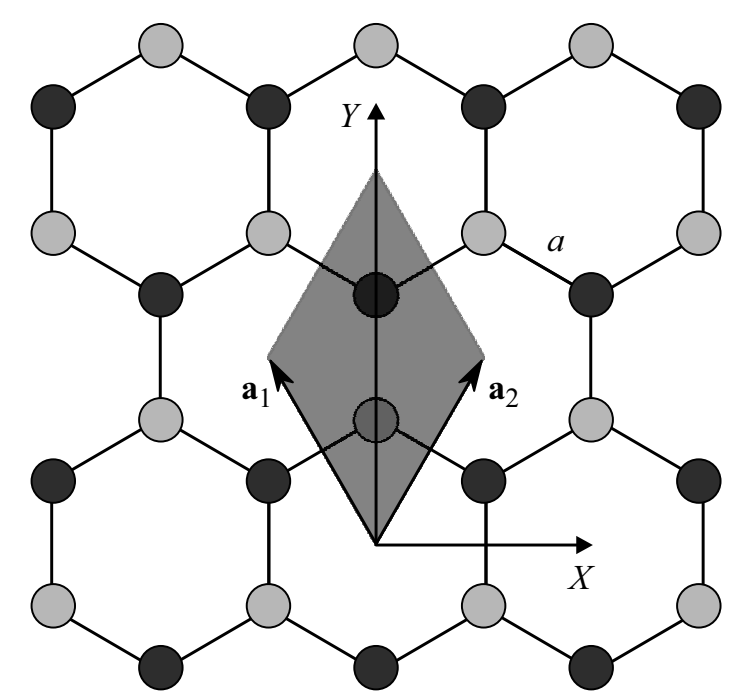

Pис. 2. Изображение гексагональной решетки графена. Серым цветом показана элементарная ячейка, в которой находятся 2 атома углерода. Светлым и темным цветом показаны узлы двух разных подрешеток кристалла. $\mathbf{a}_{1}$ и $\mathbf{a}_{2}-$ векторы трансляций решетки.

\section{2. Скалярная модель колебаний кристаллической решетки графена}

Кристаллическая решетка графена является двумерной гексагональной решеткой, представляющей собой плоскость, состоящую из шестиугольных ячеек, в вершинах которых находятся атомы углерода. Для такой решетки известно, что ее обратная решетка также будет гексагональной, а зона Бриллюэна имеет форму правильного шестиугольника. В элементарной ячейке решетки графена находятся два атома. Каждый из этих атомов при сдвиге на вектора трансляций $\mathbf{a}_{1,2}$ образует подрешетку из эквивалентных ему атомов, расположенных в эквивалентных узлах кристалла, то есть свойства кристалла независимы от точек наблюдения. На рис. 2 представлены эти две подрешетки атомов, закрашенных разными цветами. Выберем вектора трансляции решетки $\mathbf{a}_{1}$ и $\mathbf{a}_{2}$ так, как показано на рис. 2. Вектора трансляции связаны с расстоянием $a$ между ближайшими атомами углерода в вершинах шестиугольников следующим образом:

$$
\mathbf{a}_{1}=\frac{3 a}{2} \mathbf{e}_{x}+\frac{\sqrt{3} a}{2} \mathbf{e}_{y}, \quad \mathbf{a}_{2}=\frac{3 a}{2} \mathbf{e}_{x}-\frac{\sqrt{3} a}{2} \mathbf{e}_{y},
$$

где $\mathbf{e}_{x}$ и $\mathbf{e}_{y}$ - единичные орты вдоль осей $X$ и $Y$ соответственно.

На элементарную ячейку графена приходятся шесть колебательных степеней свободы - три акустических ветви (LA, TA, ZA) и три оптических (LO, TO, ZO). Здесь L - обозначает продольные фононы (волновой вектор q направлен вдоль направления поляризации), a T - поперечные (перпендикулярные направлению 


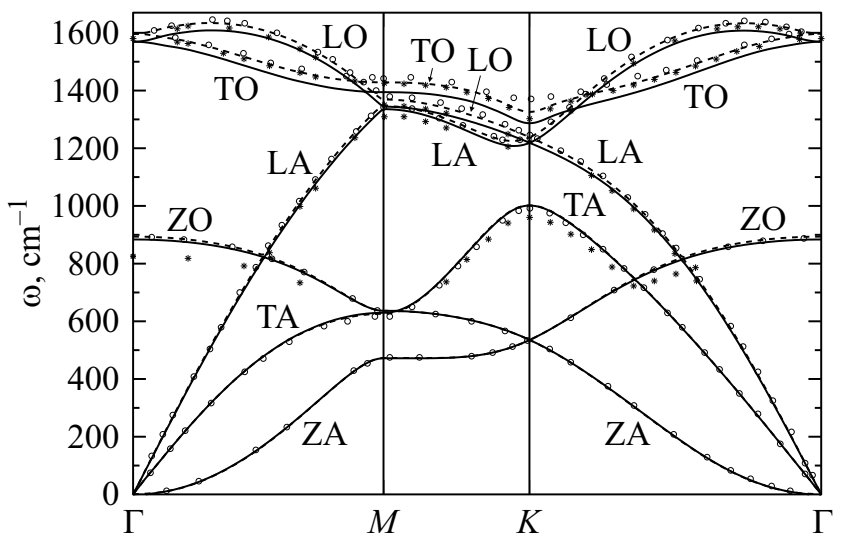

Рис. 3. Колебательные моды в графене [29].

поляризации). Две акустических моды (LA, TA) и две оптических моды (LO, TO) поляризованы в плоскости листа графена, и мы их будем называть фононными модами, а две моды (ZA, ZO) поляризованы в направлении перпендикулярном плоскости листа графена. Это так называемые изгибные моды. Они всегда являются поперечными. Принято считать, что изгибные моды имеют квадратичный закон дисперсии $\omega=\beta q^{2}$, как у электронов или магнонов, в отличие от обычного линейного закона дисперсии фононов $\omega=v_{s} q$, где $v_{s}-$ скорость звука. Однако это не является распространенной точкой зрения. Необходимы дальнейшие экспериментальные и теоретические исследования. В силу симметрии, изгибные моды не взаимодействуют с модами, поляризованными в плоскости листа графена. Последние невырождены (за исключением особых точек в зоне Бриллюэна). Их скорость распространения и частота $\omega$ зависят от направления волнового вектора q. Но при малых частотах в рамках теории упругости скорость звука $v_{s}$ не зависит от направления и графен ведет себя как однородная, изотропная сплошная среда. На рис. 3 показан колебательный спектр графена, взятый из работы [29].

Для изучения влияния беспорядка на колебательные моды в графене мы будем использовать простую скалярную модель. В этой модели смещения атомов из положения равновесия являются не векторными, а скалярными величинами. Тогда у каждого атома в графене имеются не три степени свободы, а всего лишь одна, и на два атома в элементарной ячейке приходится всего две колебательных степени свободы - акустическая и оптическая. При этом число связей на эти две степени свободы в приближении ближайших соседей равняется трем, что больше числа степеней свободы. Следовательно, согласно критерию Максвелла [30], такая скалярная модель является устойчивой. На такой упрощенной, и, как мы покажем, точно решаемой модели для изгибных и фононных мод, мы и будем изучать влияние беспорядка на колебательные моды в графене.
Скалярная модель кристаллов с беспорядком в распределении силовых констант (force constant disorder) изучалась нами и ранее в трехмерных [31,32] и двумерных [33] кристаллических решетках со случайными связями (пружинками). Полученные результаты, как мы проверяли, качественно не меняются при переходе к векторной модели [34]. Таким образом, можно утверждать, что скалярная модель, будучи намного проще, качественно правильно описывает ситуацию. Именно в этом неточном, качественном значении она и будет нами использована для изучения аморфного графена. Следует отметить, что скалярная модель вообще достаточно часто используется в физике колебаний для исследования бозонного пика в стеклах, чтобы без затрат на большие компьютерные ресурсы прийти к выводам, которые сохраняются и в векторной модели $[22,35,36]$.

\section{3. Закон дисперсии фононов поляризованных в плоскости листа графена}

Если не делать различие между L и $\mathrm{T}$ планарными фононами, то можно найти аналитически (а скалярная модель это позволяет) закон дисперсии фононов, поляризованных в плоскости листа графена. Для этого, как можно показать, достаточно учесть взаимодействие атомов в рамках первой конфигурационной окружности, учитывая только три положительных пружинки. В скалярной модели величина смещения атома из положения равновесия не зависит от его направления. Однако все остальные величины сохраняют свой векторный характер. Это, например, волновой вектор q и вектора трансляций $\mathbf{a}_{1}$ и $\mathbf{a}_{2}$.

Запишем уравнение колебаний атомов графена в гармоническом приближении для каждого сорта атомов (в каждой из подрешеток). Скалярное смещение атомов разного сорта подрешеток из положения равновесия обозначим через $U_{n k}$ и $V_{n k}$, где индексы $n, k$ нумеруют положение атомов на плоской гексагональной решетке по горизонтали и по вертикали. Учитывая, что каждый атом графена окружен тремя ближайшими соседями атомов другого сорта, уравнения движения в скалярной модели (в приближении лишь ближайших соседей) примут следующий вид:

$$
\begin{aligned}
& m \ddot{U}_{n k}=E_{0}\left(V_{n k}+V_{n-1 k}+V_{n k-1}-3 U_{n k}\right), \\
& m \ddot{V}_{n k}=E_{0}\left(U_{n k}+U_{n+1 k}+U_{n k+1}-3 V_{n k}\right),
\end{aligned}
$$

где $m$ - масса частицы (все массы частиц одинаковые), $E_{0}$ - жесткость скалярной связи-пружинки между ближайшими соседними частицами. Найдем решение этих уравнений в виде плоской волны с частотой $\omega$ и волновым вектором q:

$$
\begin{aligned}
U_{n k}^{\mathbf{q}} & =U^{0} e^{i \mathbf{q}\left(n \mathbf{a}_{1}+k \mathbf{a}_{2}\right)-i \omega t}, \\
V_{n k}^{\mathbf{q}} & =V^{0} e^{i \mathbf{q}\left(n \mathbf{a}_{1}+k \mathbf{a}_{2}\right)-i \omega t},
\end{aligned}
$$


где $U^{0}, V^{0}$ - некоторые амплитуды смещений. Тогда

$$
\begin{gathered}
U_{n+1 k}=U_{n k} e^{i \mathbf{q} \mathbf{a}_{1}}, \quad U_{n k+1}=U_{n k} e^{i \mathbf{q} \mathbf{a}_{2}}, \\
V_{n-1 k}=V_{n k} e^{-i \mathbf{q} \mathbf{a}_{1}},
\end{gathered}
$$

$\mathrm{C}$ учетом этого, уравнения колебаний принимают следующий вид:

$$
\begin{gathered}
-\omega^{2} U_{n k}=\Omega^{2}\left(V_{n k}+V_{n k} e^{-i \mathbf{q} \mathbf{a}_{1}}+V_{n k} e^{-i \mathbf{e} \mathbf{a}_{2}}-3 U_{n k}\right), \\
-\omega^{2} V_{n k}=\Omega^{2}\left(U_{n k}+U_{n k} e^{i \mathbf{q}_{1}}+U_{n k} e^{i \mathbf{e} \mathbf{a}_{2}}-3 V_{n k}\right),
\end{gathered}
$$

где $\Omega=\sqrt{E_{0} / m}$ - характерная частота колебаний одной пружинки.

Полученные уравнения имеют нетривиальное решение, когда определитель системы равен нулю. Поэтому определяющее уравнение имеет вид

$$
\Omega^{4}\left|1+e^{i \mathbf{q} \mathbf{a}_{1}}+e^{i \mathbf{q} \mathbf{a}_{2}}\right|^{2}-\left(\omega^{2}-e \Omega^{2}\right)^{2}=0 .
$$

При извлечении квадратного корня появляются два решения. Решение со знаком $(-)$ соответствует акустическим колебаниям $\omega_{a}$, решение со знаком $(+)$ соответствует оптическим колебаниям $\omega_{0}$. С учетом формулы Эйлера $2 \cos (x)=e^{i x}+e^{-i x}$, получим окончательное выражение для закона дисперсии фононов, поляризованных в плоскости листа графена в скалярной модели в приближении трех ближайших соседей и пренебрегая всеми остальными вкладами других атомов

$$
\omega(\mathbf{q})=\Omega \sqrt{(3 \pm \sqrt{3+2 f(\mathbf{q})})},
$$

где функция $f(\mathbf{q})$ определяется выражением

$$
f(\mathbf{q})=\cos \left(\mathbf{a}_{1} \mathbf{q}\right)+\cos \left(\mathbf{a}_{2} \mathbf{q}\right)+\cos \left(\left(\mathbf{a}_{1}-\mathbf{a}_{2}\right) \mathbf{q}\right) .
$$

Заметим, что полученные выражения совпадают с законом дисперсии электронов и дырок в графене $[37,38])$. Далее для удобства будем считать в относительных единицах $\Omega=1$.

Закон дисперсии фононов (10) для кристаллического графена определяет его так называемые особые точки. Более подробно они будут описаны в следующем разделе.

\section{4. Сингулярности ван-Хова и точки Дирака}

Плотность колебательных состояний $g(\omega)$ двумерных систем связана с законом дисперсии $\omega(\mathbf{q})$ следующим образом:

$$
g(\omega)=\frac{1}{4 \pi^{2}} \int_{-\pi}^{\pi} \int_{-\pi}^{\pi} \delta(\omega-\omega(\mathbf{q})) d q_{x} d q_{y} .
$$

Используя полученный в предыдущей главе закон дисперсии (10) для мод, поляризованных в плоскости листа

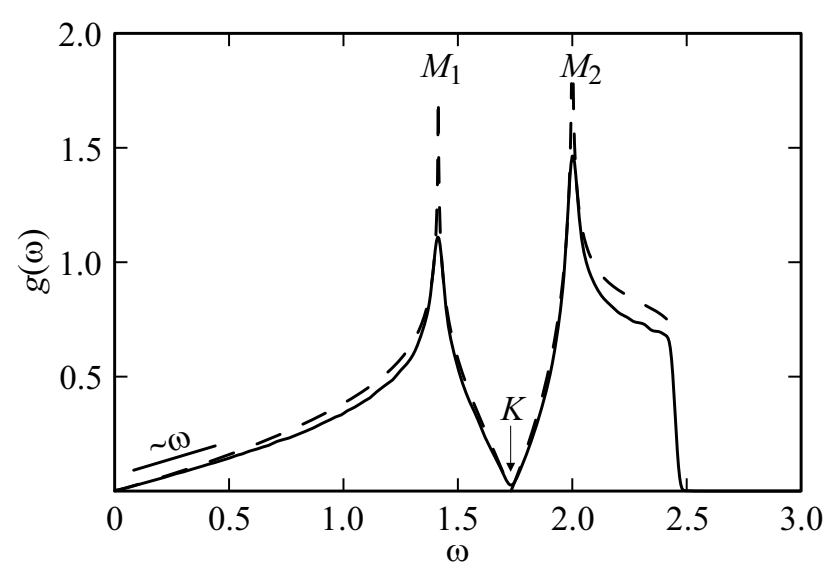

Рис. 4. Плотность колебательных состояний $g(\omega)$-мод, поляризованных в плоскости кристаллического графена, в скалярной модели. Сплошной линией показан результат численного расчета собственных значений динамической матрицы $\hat{C}_{p}$ для системы $N=1000^{2}$ атомов. Штриховой линией отмечено точное аналитическое решение (13).

графена, можно получить их плотность колебательных состояний в явном аналитическом виде [39]:

$$
\begin{aligned}
& g(\omega)= \\
& =\left\{\begin{array}{l}
\frac{\omega \sqrt{3-\omega^{2}}}{\pi^{2}} K\left(\frac{\omega}{4} \sqrt{\frac{\left(4-\omega^{2}\right)^{3}}{3-\omega^{2}}}\right), \quad 0 \leq \omega \leq \sqrt{2} \\
\frac{4\left(3-\omega^{2}\right)}{\pi^{2} \sqrt{\left(4-\omega^{2}\right)^{3}}} K\left(\frac{4}{\omega} \sqrt{\frac{3-\omega^{2}}{\left(4-\omega^{2}\right)^{3}}}\right), \quad \sqrt{2} \leq \omega \sqrt{3} \\
\frac{4 \omega\left(\omega^{2}-3\right)}{\pi^{2} \sqrt{\left(6-\omega^{2}\right)\left(\omega^{2}-2\right)^{3}}} K\left(4 \sqrt{\frac{\omega^{2}-3}{\left(6-\omega^{2}\right)\left(\omega^{2}-2\right)^{3}}}\right), \sqrt{3} \leq \omega \leq 2 \\
\frac{\omega \sqrt{\omega^{2}-3}}{\pi^{2}} K\left(\frac{1}{4} \sqrt{\frac{\left(6-\omega^{2}\right)\left(\omega^{2}-2\right)^{3}}{\omega^{2}-3}}\right), \quad 2 \leq \omega \leq \sqrt{6}
\end{array}\right.
\end{aligned}
$$

где функция $K(p)=\int_{0}^{1}\left[\left(1-x^{2}\right)\left(1-p^{2} x^{2}\right)\right]^{-1 / 2} d x-$ эллиптический интеграл первого рода. На рис. 4 плотность состояний (13) представлена пунктирной линией.

Другим подходом для нахождения колебательной плотности состояний является вычисление всех собственных чисел динамической матрицы $\hat{M}$, которая соответствует матрице силовых констант [40] и описывает колебания атомов. Вид динамической матрицы $\hat{M}$ зависит от типа колебаний. В частности, он оказывается разным для фононных и изгибных мод. Уравнение движения в гармоническом приближении в скалярной модели может быть записано в следующем виде:

$$
\omega_{i}^{2} u_{i}=\sum_{j} M_{i j} u_{j},
$$

где $u_{i}$ скалярное смещение атома с номером $i$. Из такого представления следует, что собственные значе- 
ния динамической матрицы $\lambda_{i}$ соответствуют квадратам собственных колебательных частот системы $\omega_{i}^{2}$.

Для кристаллического графена скалярная динамическая матрица планарных мод $\hat{C}_{p}$ была построена с учетом только ближайшего взаимодействия атомов с атомами первой координационной окружности по следующему принципу. Если атомы с индексами $i$ и $j$ ближайшие соседи, то соответствующий матричный элемент $C_{p_{i j}}$ равен -1 , что соответствует единичным упругим связям (скалярным пружинкам) между ближайшими соседними узлами. Если пара атомов $i$ и $j$ не является ближайшими соседями, то матричный элемент $C_{p_{i j}}$ равен 0 . Диагональные элементы матрицы $\hat{C}_{p}$ равны взятой со знаком минус сумме недиагональных элементов соответствующего столбца. Это приводит к равенству $\sum_{i} C_{p_{i j}}=0$, что обеспечивает важное условие трансляционной инвариантности системы. В таком построении динамическая матрица $\hat{C}_{p}$ оказывается устойчивой.

На рис. 4 сплошной линией изображена плотность фононных остояний, полученная путем численной диагонализации скалярной динамической матрицы кристаллического графена для планарных мод (в приближении трех пружинок) $\hat{C}_{p}$. Общее число атомов в системе $N=1000^{2}$, распределение собственных чисел матрицы $\hat{C}_{p}$ было получено с помощью КР-метода [41,42]. Можно уточнить расчет, приняв во внимание следующие координационные окружности решетки графена, однако это не дает новых качественных результатов.

При низких частотах $q a \ll 1$ плотность состояний $g(\omega) \propto \omega$ (рис. 4). Это означает, что низкочастотная плотность состояний обусловлена акустическими фононами с линейным законом дисперсии $\omega=v q$, где $v-$ скорость звука в скалярной модели. Данный закон дисперсии фононов справедлив в пространстве любой размерности и при использовании любого числа координационных окружностей (при условии, что они будут давать вклад одного знака). И действительно, как мы уже отмечали, у графена есть в колебательном спектре две таких акустических моды (TA и LA), поперечная и продольная, описывающие колебания атомов в плоскости листа графена [29]. Эти скалярные (в нашем случае) фононы с линейным законом дисперсии и описывает наша скалярная модель.

При частотах $\omega_{\mathrm{M}_{1}}=\sqrt{2}$ и $\omega_{\mathrm{M}_{2}}=2$ в плотности состояний появляются пики логарифмической расходимости (рис. 4). Это так называемые сингулярности ван-Хова, соответствующие обращению в ноль производной $d \omega / d q$. Они отвечают двум (на самом деле двенадцати) седловым точкам $M$ функций $\omega_{a, o}(\mathbf{q})$ на границе зоны Бриллюэна (в середине каждой шестиугольной стороны). Частоте $\omega_{\mathrm{M}_{1}}$ соответствует логарифмическая сингулярность ван-Хова от акустической ветви, частоте $\omega_{\mathrm{M}_{2}}-$ от оптической ветви. Существование этих функций неявно означает, что в отсутствие беспорядка волновой вектор фонона q является хорошим квантовым числом, и представление о фононах как квазичастицах имеет физический смысл. Резкий обрыв до нуля плотности состояний в конце спектра на частоте $\omega_{\max }=\sqrt{6}$ это третья сингулярность ван-Хова, связанная с максимумом функции $\omega_{o}(\mathbf{q})$ оптических колебаний при $\mathbf{q}=0$.

Также на рис. 4 видно, что при некоторой частоте $\omega=\omega_{K}$ плотность состояний $g(\omega)$ обращается в ноль. Это так называемая точка Дирака. Как известно, зона Бриллюэна кристаллического графена имеет форму правильного шестиугольника. Поскольку массы атомов в обеих подрешетках одинаковы, то в шести точках Дирака K [37] (вершинах этого шестиугольника), на границе зоны Бриллюэна, плотность состояний $g(\omega)$ имеет $\mathrm{V}$-образную форму и линейно по частоте $\omega$ обращается в ноль. Это означает, что закон дисперсии фононов в окрестности этих точек, как и в начале координат, тоже линейный и имеет вид $\omega=\omega_{\mathrm{K}} \pm v_{\mathrm{K}}\left|\mathbf{q}-\mathbf{q}_{\mathrm{K}}\right|$, где $v_{\mathrm{K}}-$ скорость этих фононов. Изочастотные поверхности в точках Дирака $\mathrm{K}$ имеют форму конуса (конус Дирака). Конус оптических фононов направлен вниз, а акустических вверх, так что конусы (их всего 6) имеют общую вершину.

\section{5. Изгибные моды}

Чтобы найти закон дисперсии изгибных мод, необходимо правильно написать их волновое уравнение, и, как следствие, динамическую матрицу изгибных мод. В макроскопической теории упругости изгибных мод тонких пластинок показывается, что волновое уравнение содержит квадрат оператора Лапласа $\Delta^{2}[28]$, который играет роль динамической матрицы в низкочастотной области спектра. Поскольку дискретному лапласиану $\Delta$ на решетке графена соответствует динамическая матрица планарных мод $\hat{C}_{p}$, то можно предполагать, что динамической матрицей изгибных мод является $\hat{C}_{f}=\hat{C}_{p}^{2}$. Это предположение между тем нуждается в проверке. И мы покажем ниже, что оно действительно при определенных условиях имеет место.

В такой матрице изгибных мод $\hat{C}_{f}$, построенной на матрице трех ближайших связей $\hat{C}_{p}$, пружинки (связи) размещены не только между ближайшими соседями, но и между соседями соседей. Другими словами, вокруг каждого атома имеется не три единичных пружинки, а девять. Легко проверить, возводя известную матрицу $\hat{C}_{p}$ в квадрат, что знак дополнительных 6 пружинок оказывается не положительным, а отрицательным. Их величина в 6 раз меньше величины трех положительных пружинок. Отрицательные пружинки говорят о близкой неустойчивости в системе, которая возникает в случае, если отрицательные пружинки по модулю превысят одну шестую положительных пружинок (см. Приложение). Об этом же говорят численные результаты по расчету модуля Юнга матрицы $\hat{C}_{f}$. Модуль Юнга в термодинамическом пределе оказывается равным нулю. 
Такой подход приводит к закону дисперсии изгибных мод в графене в виде

$$
\omega(\mathbf{q}=\tilde{\Omega})(3 \pm \sqrt{3+2 f(\mathbf{q})}),
$$

где функция $f(\mathbf{q})$ по-прежнему определяется выражением (11). Согласно макроскопической теории упругости, низкочастотные изгибные моды тонких пластинок и стержней имеют закон дисперсии $\omega \propto q^{2}$ [28]. Действительно, из полученного закона дисперсии изгибных мод (15) следует, что при малых $q a \ll 1$ частота акустической и оптической ветвей $\omega_{a, o} \propto q^{2}$. При этом закон дисперсии (15) по-прежнему определяет особые точки графена М и К, а результаты его расчета для изгибных мод имеют хорошее согласие с данными работы [34]. Согласие двух кривых настолько хорошее, что ставит вопрос о физической причине этого совпадения (рис. 5). Одно из предположений заключается в том, что формула (15) действительно является точной и операция возведения матрицы $\hat{C}_{p}$ в квадрат имеет большие основания, чем кажется на первый взгляд (см. Приложение).

Используя закон дисперсии (15) для изгибных мод листа графена, можно получить их плотность состояний в явном аналитическом виде [39]:

$$
g(\omega)=\left\{\begin{array}{l}
\frac{\sqrt{3-\omega}}{2 \pi^{2}} K\left(\frac{1}{4} \sqrt{\omega \frac{(4-\omega)^{3}}{3-\omega}}\right), \quad 0 \leq \omega \leq 2 \\
\frac{2(3-\omega)}{\pi^{2} \sqrt{\omega(4-\omega)^{3}}} K\left(4 \sqrt{\frac{3-\omega}{\omega(4-\omega)^{3}}}\right), \quad 2 \leq \omega \leq 3 .
\end{array}\right.
$$

На рис. 6 формула (16) представлена пунктирной линией.

Так же на рис. 6 изображена плотность состояний изгибных колебаний, полученная путем численной диагонализации динамической матрицы $\hat{C}_{f}$. Общее число атомов в системе $N=1000^{2}$, распределение собственных чисел матрицы $\hat{C}_{f}$ было получено с помощью КР метода $[41,42]$.

При низких частотах $g(\omega) \propto$ const, что согласуется с законом дисперсии изгибных мод $\omega \propto \mathbf{q}^{2}$. Также из рис. 6 видно, что все особые точки сохраняют свои свойства. Это позволяет говорить о том, что представленная динамическая матрица $\hat{C}_{f}$ качественно правильно описывает низкочастотные изгибные моды кристаллического графена.

Таким образом, скалярная модель дает результаты, согласующиеся с результатами других более сложных моделей [29,34], и качественно правильно описывает основные структурные особенности графена. Возникает правомерный вопрос - как плотность состояний $g(\omega)$ будет выглядеть в аморфном графене, то есть при наличии беспорядка? Естественно предположить, что две логарифмические сингулярности ван-Хова должны с ростом беспорядка ушириться по частоте, слиться друг с другом и плавно перейти в один бозонный пик. Однако из-за наличия в кристалле графена двух типов фононов, акустических и оптических, не ясно, с какими фононами

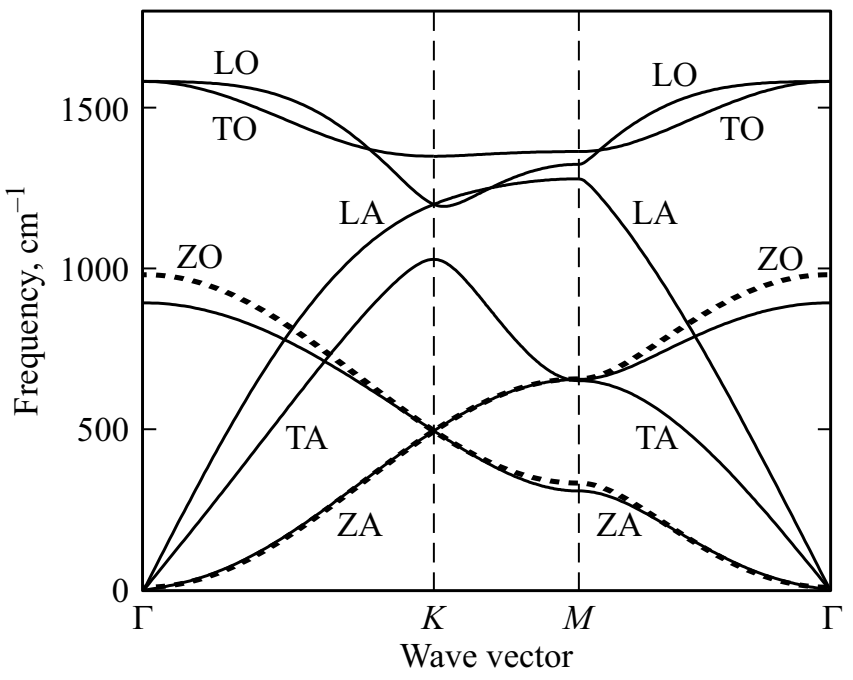

Рис. 5. Сравнение закона дисперсии фононных мод в графене из работы [34] с результатом (15) для Z-- и ZА-фононов, обозначенным пунктирной линией.

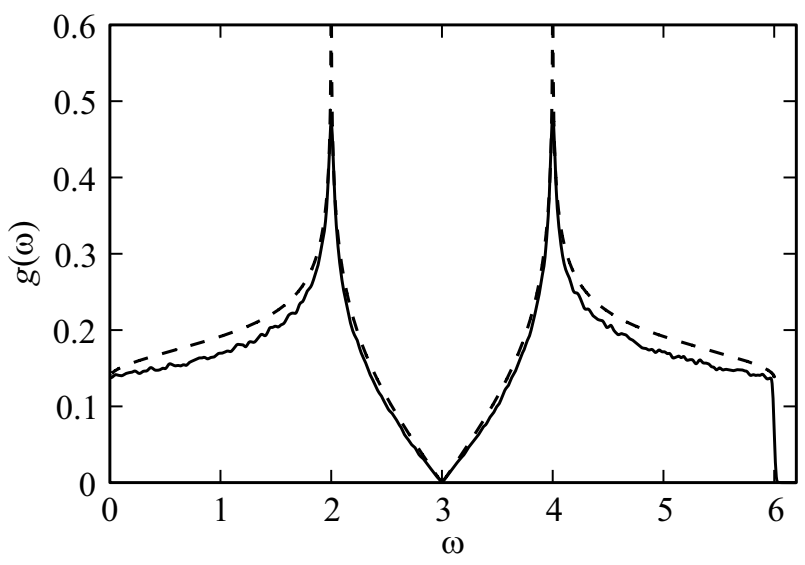

Рис. 6. Плотность колебательных состояний $g(\omega)$ изгибных мод кристаллического графена в скалярной модели. Сплошной линией показан результат численного расчета собственных значений динамической матрицы $\hat{C}_{f}$ для системы $N=1000^{2}$ атомов. Штриховой линией отмечено точное аналитическое решение (16).

будет связан бозонный пик. В следующих разделах мы попытаемся ответить на эти вопросы.

\section{6. Модель устойчивых случайных матриц}

Вблизи положения устойчивого равновесия энергия взаимодействия атомов $U$ представима в виде суммы квадратичных по смещениям $u_{i}$ форм [32]:

$$
U=\frac{1}{2} \sum_{k=1}^{K}\left(\sum_{i=1}^{N} A_{i k} u_{i}\right)^{2}
$$

В скалярной модели смещения $u_{i}$ являются скалярными величинами, поэтому число степеней свободы системы 
равно числу атомов $N$. Индекс $k=1,2, \ldots, K$ нумерует „связи“ атомов в системе, энергия которых имеет вид положительно определенной квадратичной формы с коэффициентами $A_{i k}$. Матрица $\hat{A}$ является в общем случае прямоугольной матрицей размером $N \times K$. При этом для устойчивости всей системы необходимо, чтобы число ее связей $K$ было не меньше числа ее степеней свободы $N$ [30], поэтому $K \geq N$.

Динамическая матрица $\hat{\hat{M}}$ в случае одинаковых единичных масс $m_{i}=1$ связана с энергией взаимодействия $U$ следующим образом:

$$
M_{i j}=\frac{\partial^{2} U}{\partial u_{i} \partial u_{j}} .
$$

Из формул (17) и (18) следует, что в матричной записи динамическая матрица $\hat{M}$ имеет вид

$$
\hat{M}=\hat{A} \hat{A}^{T} .
$$

Из представления (19) следует, что матрица $\hat{M}$ является квадратной, симметричной матрицей $N \times N$, поэтому все ее собственные числа $\lambda_{i}$ вещественны. Кроме этого, все $\lambda_{i}$ неотрицательны, поскольку равны квадратам сингулярных чисел матрицы $\hat{A}$. Такое условие на собственные числа $\hat{M}$ обеспечивает важное требование механической устойчивости системы, так как $\lambda_{i}$ должны соответствовать квадратам собственных колебательных частот $\omega_{i}^{2}$.

Вследствие локального беспорядка аморфной системы можно предполагать, что матрица $\hat{A}$ должна носить некоторый неупорядоченный характер. Поэтому рассмотрим матрицу $\hat{A}$ как случайную квадратную матрицу, построенную на двумерной решетке графена с учетом только ближайшего взаимодействия по следующему принципу. Если атомы с индексами $i$ и $j$ ближайшие соседи, то соответствующий матричный элемент $A_{i j}$ есть случайное число, распределенное по Гауссу с единичной дисперсией $V^{2}=1$ и средним значением равным нулю. Если атомы $i$ и $j$ не являются ближайшими соседями, то матричный элемент $A_{i j}=0$. Диагональные элементы матрицы $\hat{A}$ равны взятой со знаком минус сумме недиагональных элементов соответствующего столбца матрицы $\hat{A}$. Это приводит к равенству $A_{i j}=0$, что обеспечивает важное условие трансляционной инвариантности системы.

Оказывается, что построенная таким образом случайная динамическая матрица $\hat{M}=\hat{A} \hat{A}^{T}$ приводит к нулевой макроскопической жесткости всей системы $[31,33,43]$. Это связано с тем, что число степеней свободы $N$ (число строк матрицы $\hat{A}$ ) равно числу связей $K$ (числу столбцов матрицы $\hat{A})$, так как по описанному выше построению матрица $\hat{A}$ является квадратной [30]. В такой предельно мягкой системе акустические фононы не распространяются, так как скорость звука в ней равна нулю.

Добавление новых связей делает модуль Юнга системы $E$ отличным от нуля, и в такой системе могут распространятся фононы. Число связей можно увеличить, например, следующими способами.
Первый способ. Добавим к случайной квадратной матрице $\hat{A} \hat{A}^{T}$, обладающей нулевой жесткостью и отвечающей за беспорядок в системе, регулярную матрицу $\mu \hat{C}_{p}$ планарных мод, отвечающую за порядок

$$
\hat{M}=\hat{A} \hat{A}^{T}+\mu \hat{C}_{p} .
$$

Физический смысл такого суммирования заключается в том, что недиагональные элементы матрицы $\hat{C}_{p}$ и матрицы $\hat{A} \hat{A}^{T}$ представляют собой коэффициенты упругости $k_{i j}$ параллельно соединенных упругих пружинок (взятые со знаком минус). А при параллельном соединении пружинок, как известно, их коэффициенты жесткости складываются.

Параметр системы $\mu$, характеризующий жесткость отдельной пружинки, отвечает за степень порядка в системе и меняется в интервале $0 \leq \mu<\infty$. Случай $\mu \gg 1$ соответствует системе с небольшими относительными флуктуациями связей, т.е. слабому беспорядку. В этом случае второе слагаемое в формуле (20) значительно больше первого, и модуль Юнга системы $E$ зависит от параметра $\mu$ как $E \propto \mu$. Однако наибольший интерес представляет противоположный предельный случай $\mu \ll 1$, который соответствует аморфному телу с большими флуктуациями, т.е. сильному беспорядку. В этом случае модуль Юнга системы $E$ зависит от параметра $\mu$ как $E \propto \sqrt{\mu}[31-33]$.

Второй способ. Для планарных мод. Рассмотрим две случайные матрицы $\hat{A}_{1}$ и $\hat{A}_{2}$ размером $N \times N$, построенные по такому же принципу, что и случайная квадратная матрица $\hat{A}$. Будем добавлять случайным образом в $\hat{A}_{1}$ некоторую долю столбцов $\hat{A}_{2}$. Полученная таким образом новая прямоугольная матрица $\hat{B}$ описывает систему, у которой число связей (число столбцов $K$ ), больше числа степеней свободы (числа строк $N$ ). Это означает, что система $\hat{B} \hat{B}^{T}$ обладает отличной от нуля жесткостью. Параметр $\varkappa$ характеризует относительную долю добавленных столбцов как $\varkappa=K / N-1$. Таким способом можно получить значения $\varkappa$ в диапазоне $0 \leq \varkappa \leq 1$. При $\varkappa \ll 1$ модуль Юнга $E \propto \varkappa$.

Для получения больших значений $x$ необходимо повторить описанную выше процедуру добавления столбцов, взяв в качестве „стэковых“ матриц полученную прямоугольную матрицу $\hat{B}$ и другую реализацию матрицы $\hat{A}$. Полученная таким способом динамическая матрица $\hat{M}$ при $n \rightarrow \infty$ стремится к кристаллической матрице $\sqrt{\varkappa} \hat{C}_{p}$. При $\varkappa \gg 1$ (т.е. при слабом беспорядке) модуль Юнга увеличивается с ростом $\varkappa$ по закону $E \propto \sqrt{\varkappa}[33,44]$.

\section{7. Бозонный пик}

Увеличение числа связей приводит к тому, что в системе появляются упругие колебания - фононы. На рис. 7 показан результат численного расчета плотности колебательных состояний $g(\omega)$ для двух способов увеличения числа связей при различных параметрах систе- 
мы $\mu$ и $\varkappa$. Как видно, две совершенно разных модели случайных матриц обладают схожими колебательными свойствами.

В низкочастотной области плотности состояний наблюдается дебаевский (фононный) вклад $g(\omega) \propto \omega$. При достаточно слабом беспорядке в системе $\mu, \varkappa \gg 1$ наблюдаются два высокочастотных пика, обусловленных сингулярностями ван-Хова в спектре кристаллического графена, показанных на рис. 4. Однако, как видно из рис. 7, по мере увеличения беспорядка в системе эти пики размываются, растут по величине и сдвигаются влево, в область более низких частот. При этом правый пик, обусловленный оптическими фононами, быстро уменьшается по амплитуде и сливается с пиком слева, обусловленным акустическими фононами. При частотах $\omega \simeq \sqrt{\mu}$ (или $\omega \simeq \varkappa$ ) наблюдается только один низкочастотный пик в приведенной плотности состояний $g(\omega) / \omega$ бозонный пик.

Для расчета плотности состояний изгибных колебаний в аморфном графене будем использовать ансамбль матриц, аналогичный форме (20), с динамической матрицей кристалла $\hat{C}_{f}$ :

$$
\hat{M}=\hat{A} \hat{A}^{T}+\mu \hat{C}_{f}
$$

Результат численных расчетов показан на рис. 8 .
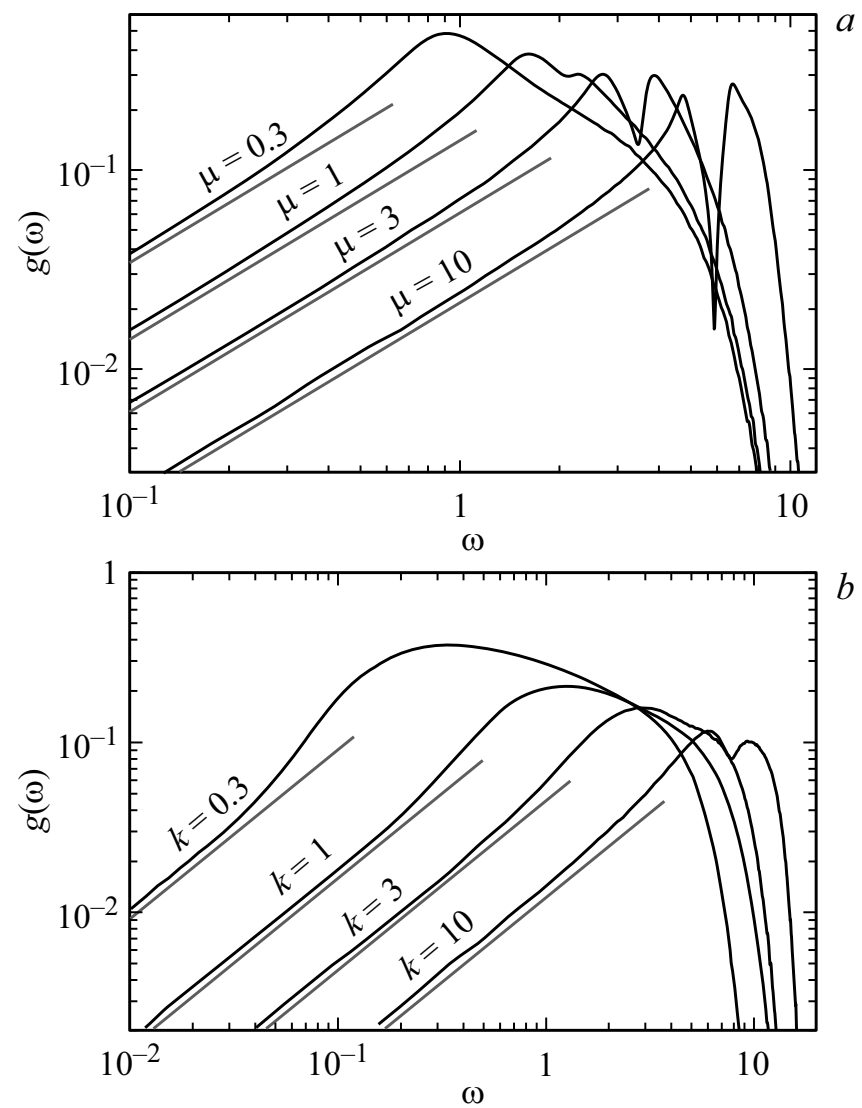

Рис. 7. Плотность колебательных состояний скалярной модели аморфного графена для разных параметров системы $\mu(a)$ и $\varkappa(b)$. Прямыми линиями отмечен дебаевский фононный вклад $g(\omega) \propto \omega$.
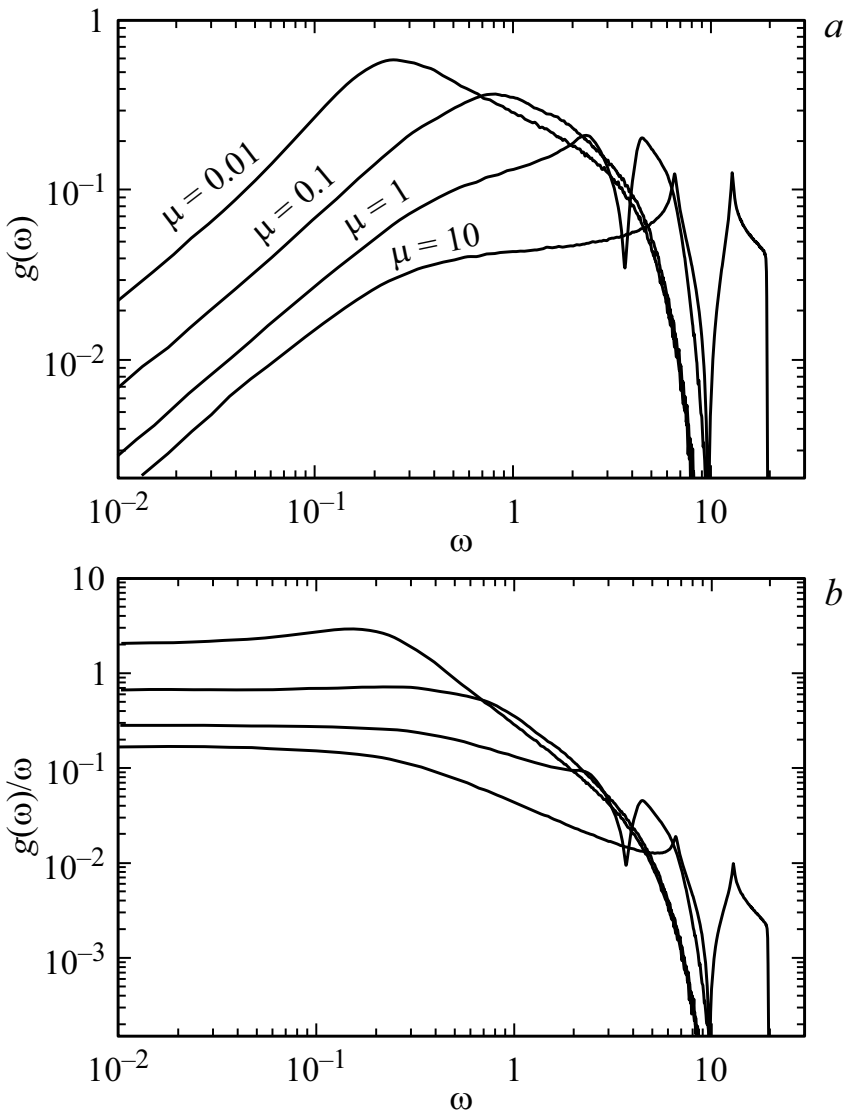

Рис. 8. $a-$ плотность колебательных состояний $g(\omega)$ динамической матрицы $\hat{M}=\hat{A} \hat{A}^{T}+\mu \hat{C}_{f}$ для различных параметров системы $\mu ; b-$ приведенная плотность колебательных состояний $g(\omega) / \omega$.

Видно, что низкочастотная область плотности состояний имеет зависимость $g(\omega) \propto \omega$. Это означает, что при добавлении даже малого беспорядка в системе появляются фононы с законом дисперсии $\omega \propto q$. Их появление связано с тем, что в выражении (21) каждое из слагаемых описывает систему с нулевой макроскопической жесткостью, находящуюся по критерию Максвелла на пороге устойчивости $N=K$. Суммирование приводит к увеличению числа связей $K$ в 2 раза, а число степеней свободы $N$ оставляет прежним. В результате, модуль Юнга системы не равен нулю, и при малых частотах вместо изгибных мод в системе распространяются фононы.

При достаточно слабом беспорядке в системе $\mu \gg 1$ наблюдаются два высокочастотных пика, обусловленных сингулярностями ван-Хова для оптических и акустических фононов. Затем отчетливо наблюдается небольшое плато, связанное с законом дисперсии изгибных мод $\omega \propto q^{2}$. Однако по мере увеличения беспорядка $\mu \ll 1$ пики сингулярностей ван-Хова пропадают, и возникает бозонный пик. При этом наблюдается переходное состояние системы, в котором и пики сингулярностей ван-Хова, и бозонный пик отсутствуют (на рис. 8 этому случаю соответствует значение параметра $\mu=0.1$ ). 


\section{8. Заключение}

Используя модель устойчивых случайных матриц, обладающих трансляционной симметрией, мы показали, что в аморфном графене, при достаточно сильном беспорядке в распределении силовых констант может наблюдаться только один бозонный пик в приведенной плотности состояний $g(\omega) / \omega$. Напротив, при относительно слабом беспорядке мы имеем два пика в плотности состояний, соответствующих сингулярностям ван-Хова для акустических и оптических фононов кристаллического графена. Эти два пика говорят о том, что волновой вектор q в этой области частот является хорошим квантовым числом. С ростом беспорядка пики смещаются влево, в область малых частот, и постепенно сливаются в один бозонный пик, который далее смещается в область все более низких частот по мере роста беспорядка в системе. Причем первыми за счет беспорядка разрушаются оптические фононы, а уже потом акустические. Как и в случае решеток с одним атомом в элементарной ячейке, при наличии только одного пика, бозонного, фононы как квазичастицы существуют только при частотах ниже частоты бозонного пика. Справа от бозонного пика собственным модам можно приписывать частоту $\omega$, но не волновой вектор q. Однако эти аспекты мы собираемся рассмотреть в отдельной работе.

Также в работе отдельно были рассмотрены изгибные моды графена, для которых плотность состояний при увеличении беспорядка из пиков сингулярностей ван-Хова переходит в бозонный пик. Однако существует диапазон значений параметра $\mu$, при которых пики сингулярностей ван-Хова уже исчезли, а бозонный пик еще не возник. При этом изгибные моды с плотностью состояний $g(\omega) \propto$ const уступают обычным фононам. В связи с этим, можно предполагать, что формирование бозонного пика скорее связано не с сингулярностями ван-Хова, а с наличием в системе низкочастотных фононов с дебаевской плотностью состояний $g(\omega) \propto \omega$.

Кроме этого, мы показали, что изгибные моды разрушаются даже при относительно слабом беспорядке в системе, и в аморфном графене доминирующую часть низкочастотного спектра занимают фононы. Это, в свою очередь, означает, что наблюдать изгибные моды на малых частотах трудно или даже невозможно. Это важный результат нашей теории изгибных мод.

\section{Приложение}

Рассмотрим по-отдельности связанные колебания „темных“ и „светлых“ атомов решетки графена, изображенных на рис. 2,c амплитудами $V_{n, m}$ и $U_{n, m}$ соответственно. Индексы $n$ и $m$ нумеруют положение атома вдоль направлений векторов трансляций $\mathbf{a}_{1}$ и $\mathbf{a}_{2}$. Пусть $\varkappa_{1}$ и $\varkappa_{2}$ упругие константы взаимодействия атома с соседями из первой и второй координационных окружностей, насчитывающих три и шесть атомов соответственно.
Считая массы атомов одинаковыми, запишем их уравнения движения

$$
\begin{aligned}
m \frac{d^{2} U_{n, m}}{d t^{2}}= & \varkappa_{1}\left(V_{n, m}+V_{n-1, m}+V_{n, m-1}-3 U_{n, m}\right) \\
& +\varkappa_{2}\left(U_{n+1, m}+U_{n-1, m}+U_{n, m+1}\right. \\
& \left.+U_{n, m-1}+U_{n-1, m+1}+U_{n+1, m-1}-6 U_{n, m}\right), \\
m \frac{d^{2} V_{n, m}}{d t^{2}}= & \varkappa_{1}\left(U_{n, m}+U_{n+1, m}+U_{n, m+1}-3 V_{n, m}\right) \\
+ & \varkappa_{2}\left(V_{n+1, m}+V_{n-1, m}+V_{n, m+1}\right. \\
+ & \left.V_{n, m-1}+V_{n-1, m+1}+V_{n+1, m-1}-6 V_{n, m}\right),
\end{aligned}
$$

Будем искать решение уравнений (22) в виде плоских бегущих волн возмущения с частотой $\omega$ и волновым вектором q:

$$
\begin{aligned}
& U_{n, m}=U^{0} e^{i \mathbf{q}\left(n \mathbf{a}_{1}+m \mathbf{a}_{2}\right)-i \omega t}, \\
& V_{n, m}=V^{0} e^{i \mathbf{q}\left(n \mathbf{a}_{1}+m \mathbf{a}_{2}\right)-i \omega t} .
\end{aligned}
$$

После подстановки (23) в уравнения движения (22), получим

$$
\begin{aligned}
-m \omega^{2} U_{n, m}= & \varkappa_{1}\left(V_{n, m} A(-\mathbf{q})-3 U_{n, m}\right) \\
& +2 \varkappa_{2} U_{n, m}(f(\mathbf{q})-3), \\
-m \omega^{2} V_{n, m}= & \varkappa_{1}\left(U_{n, m} A^{*}(-\mathbf{q})-3 V_{n, m}\right) \\
& +2 \varkappa_{2} V_{n, m}(f(\mathbf{q})-3),
\end{aligned}
$$

где для удобства введены функции

$$
\begin{gathered}
A(\mathbf{q})=\left(1+e^{i \mathbf{a}_{1} \mathbf{q}}+e^{i \mathbf{a}_{2} \mathbf{q}}\right), \\
f(\mathbf{q})=\cos \left(\mathbf{a}_{1} \mathbf{q}\right)+\cos \left(\mathbf{a}_{2} \mathbf{q}\right) \cos \left(\left(\mathbf{a}_{1}-\mathbf{a}_{2}\right) \mathbf{q}\right)
\end{gathered}
$$

Введя обозначения $\Omega_{1}^{2}=\varkappa_{1} / m$ и $\Omega_{2}^{2}=\varkappa_{2} / m$ и условно допуская, что значение $\Omega_{2}^{2}$ может быть отрицательным, перепишем систему уравнений (24) в виде

$$
\begin{gathered}
U_{n, m}\left(\omega^{2}-3 \Omega_{1}^{2}-2 \Omega_{2}^{2}(3-f(\mathbf{q}))+V_{n, m} \Omega_{1}^{2} A(\mathbf{q})=0,\right. \\
V_{n, m}\left(\omega^{2}-3 \Omega_{1}^{2}-2 \Omega_{2}^{2}(3-f(\mathbf{q}))+U_{n, m} \Omega_{1}^{2} A^{*}(\mathbf{q})=0 .\right.
\end{gathered}
$$

Уравнения (27) имеют нетривиальное решение при условии равенства нулю определителя

$$
\left(\omega^{2}-3 \Omega_{1}^{2}-2 \Omega_{2}^{2}(3-f(\mathbf{q}))^{2}-\Omega_{1}^{4} A(\mathbf{q}) A^{*}(\mathbf{q})=0 .\right.
$$

После преобразования сомножителей во втором слагаеMOM

$$
A(\mathbf{q}) A^{*}(\mathbf{q})=3+2 f(\mathbf{q}),
$$

мы получаем формулу для закона дисперсии изгибных мод в графене с учетом двух координационных окружностей

$$
\omega^{2}(\mathbf{q})=\Omega_{1}^{2}(3 \pm \sqrt{3+2 f(\mathbf{q})})+2 \Omega_{2}^{2}(3-f(\mathbf{q})) .
$$


„Критическое“ соотношение значений упругих констант $\varkappa_{1}$ и $\varkappa_{2}$ может быть получено из условия стремления к нулю частоты (30) в пределе малых значений $a q \ll 1$ для акустической ветви. Частоты оптической ветви всегда отличны от нуля, поэтому для них это условие роли не играет. В первом приближении функция $f(\mathbf{q})$ равна.

$$
f(\mathbf{q}) \approx 3-\frac{9}{4} a^{2} q^{2}+\frac{27}{64} a^{4} q^{4}
$$

В этом случае частота акустической ветви определяется соотношением

$$
\omega(\mathbf{q}) \approx \frac{3}{4}\left(\Omega_{1}^{2}+6 \Omega_{2}^{2}\right) a^{2} q^{2}-\frac{3}{64}\left(\Omega_{1}^{2}+18 \Omega_{2}^{2}\right) a^{4} q^{4} .
$$

При соотношении упругих констант $\varkappa_{2}=-\varkappa_{1} / 6, \varkappa_{2}<0$, квадратичный по $q$ член в (32) пропадает, что дает закон дисперсии изгибных мод

$$
\omega(\mathbf{q}) \approx \frac{3}{4}\left(\Omega_{1}^{2}+6 \Omega_{2}^{2}\right) a^{2} q^{2}-\frac{3}{64}\left(\Omega_{1}^{2}+18 \Omega_{2}^{2}\right) a^{4} q^{4} .
$$

Физически это понять легко. При критическом значении коэффициентов упругости $\varkappa_{2}=-\varkappa_{1} / 6$ модуль Юнга графеновой пленки на изгиб равен нулю. Изгибные фононы не могут распространятся в такой мягкой среде, где их модуль упругости и скорость звука равны нулю. Их сменяют изгибные волны с иным, более сложным законом дисперсии. Вблизи критического состояния феноменологически это можно записать в виде

$$
\omega^{2}(\mathbf{q})=a q^{2}+\beta q^{4} .
$$

где $\alpha \propto \varkappa_{2}+\varkappa_{1} / 6$, а $\beta \sim 1$. В точке изгибного перехода $\alpha=0$. Отсюда следует, что при низких частотах $\omega<\sqrt{\alpha}$ эти колебания являются фононами с линейным законом дисперсии, и скорость звука $v \propto \sqrt{\alpha}$ обращается в ноль в критической точке. При более высоких частотах это изгибные волны с квадратичным законом дисперсии. Стоит отметить, что исчезновение квадратичного по $q$ члена в законе дисперсии (32) возможно только при рассмотрении более дальних отрицательный связей.

В случае учета взаимодействия только с соседями из первой координационной окружности (что эквивалентно условию $\left.\varkappa_{2}=0\right)$, уравнение (30) сводится к соотношению

$$
\omega_{1}^{2}(\mathbf{q})=\Omega_{1}^{2}(3 \pm \sqrt{3+2 f(\mathbf{q})}) .
$$

Покажем, закон дисперсии изгибных $\omega_{f}(\mathbf{q})$ мод соответствует $\omega^{2}(\mathbf{q})$. Для этого преобразуем уравнение (30) с учетом „критического“ соотношения упругих констант $\varkappa_{2}=-\varkappa_{1} / 6$ следующим образом:

$$
\begin{aligned}
\omega_{f}^{2}(\mathbf{q}) & =\Omega_{1}^{2}\left(2 \pm \sqrt{3+2 f(\mathbf{q})}+\frac{f(\mathbf{q})}{3}\right) \\
& =\frac{\Omega_{1}^{2}}{6}(3 \pm \sqrt{3+2 f(\mathbf{q})})^{2}=\frac{1}{6 \Omega_{1}^{2}} \omega_{1}^{4}(\mathbf{q}) .
\end{aligned}
$$

Другими словами, закон дисперсии изгибных мод (36) может быть определен как квадрат закона дисперсии фононов (35) при учете первой координационной окружности. В свою очередь, это обосновывает наш выбор динамической матрицы изгибных мод $\hat{C}_{f}$, определенной как квадрат динамической матрицы планарных колебаний $\hat{C}_{p}$.

Таким образом, включение взаимодействия с атомами из второй координационной окружности коренным образом меняет закон дисперсии колебательных мод графена и в случае „критического“ соотношения упругих констант приводит к смене линейного закона дисперсии, следующего из взаимодействия с атомами первой координационной окружности, квадратичной зависимостью. Ответить на вопрос, какой же в действительности закон дисперсии у изгибных мод в графене, мы не можем. Он зависит от соотношения упругих констант $\varkappa_{1}$ и $\varkappa_{2}$, которые должны быть определены либо из эксперимента, либо из более глубокой теории. Так, если отталкиваться от эксперимента, то экспериментальные точки на рис. 3 говорят, что мы находимся либо в критической ситуации, либо очень близко к ней. Это ясно из параболического на вид закона дисперсии (понятно, что требуется более тщательная обработка экспериментальных данных). К такому же выводу мы приходим после анализа теоретических данных, показанных на рис. 5. Там тоже отчетливо видна нелинейная зависимость $\omega(q)$ в точке Г для изгибных мод ZА-типа. Но авторы этой работы признают, что они получили квадратичную зависимость путем обращения скорости звука в ноль. Также ведут себя изгибные моды и у нас. В критической точке модуль Юнга и скорость звука у нас тоже обращаются в ноль. Ясно, что требуется более подробное изучение этого вопроса для доказательства существования отрицательных пружинок в графене и закона дисперсии $q^{2}$.

\section{Благодарности}

Мы искренне благодарим Я.М. Бельтюкова и В.Л. Гуревича за полезные критические замечания и Л.Е. Голуба за ссылку [34]. Мы благодарим М.М. Глазова за интересную дискуссию по изгибным модам. Мы также особо благодарны А.С. Иоселевичу за подробное обсуждение статьи.

\section{Конфликт интересов}

Авторы заявляют, что у них нет конфликта интересов.

\section{Список литературы}

[1] A. Kumar, M. Wilson, M.F. Thorpe. J. Phys. Condens. Matter 24, 485003 (2012).

[2] R. Ravinder, R. Kumar, M. Agarwal, N.M. Anoop Krishnan. Sci. Rep. 9, 4517 (2019). 
[3] J. Kotakoski, A.V. Krasheninnikov, U. Kaiser, J.C. Meyer. Phys. Rev. Lett. 106, 105505 (2011).

[4] S. Bazrafshan, A. Rajabpour. Int. J. Heat Mass Transf. 112, 379 (2017).

[5] C. Lee, X. Wei, J.W. Kysar, J. Hone. Science 321, 385 (2008).

[6] J.S. Bunch, A.M. van der Zande, S.S. Verbridge, I.W. Frank, D.M. Tanenbaum, J.M. Parpia, H.G. Craighead, P.L. McEuen. Science 315, 490 (2007).

[7] A.K. Geim, K.S. Novoselov. Nature Mater 6, 183 (2007).

[8] A.H. Castro Neto, F. Guinea, N.M.R. Peres, K.S. Novoselov, A.K. Geim. Rev. Mod. Phys. 81, 109 (2009).

[9] V.M. Apalkov, T. Chakraborty. Phys. Rev. B 84, 033408 (2011).

[10] А.В. Елецкий, И.М. Искандарова, А.А. Книжник, Д.Н. Красиков. УФН 181, 233 (2011).

[11] A.A. Balandin, S. Ghosh, W. Bao, I. Calizo, D. Teweldebrhan, F. Miao, C.N. Lau. Nano Lett. 8, 902 (2008).

[12] D.V. Tuan, A. Kumar, S. Roche, F. Ortmann, M.F. Thorpe, P. Ordejon. Phys. Rev. B 86, 121408 (2012).

[13] C. Carpenter, D. Maroudas, A. Ramasubramaniam. Appl. Phys. Lett. 103, 013102 (2013).

[14] A. Zandiatashbar, G.-H. Lee, S.J. An, S. Lee, N. Mathew, M. Terrones, T. Hayashi, C.R. Picu, J. Hone, N. Koratkar. Nature Commun. 5, 3186 (2014).

[15] G. Lypez-Polin, C. Gymez-Navarro, V. Parente, F. Guinea, M.I. Katsnelson, F. Perez-Murano, J. Gymez-Herrero. Nature Phys. 11, 26 (2015).

[16] T. Zhu, E. Ertekin. Phys. Rev. B 93, 155414 (2016).

[17] T. Zhu, E. Ertekin. Nano Lett. 16, 4763 (2016).

[18] P.B. Allen, J.L. Feldman. Phys. Rev. B 48, 12581 (1993).

[19] P.B. Allen, J.L. Feldman, J. Fabian, F. Wooten. Phil. Mag. B 79, 1715 (1999).

[20] V.L. Gurevich, D.A. Parshin, H.R. Schober. Phys. Rev. B 67, 4203 (2003).

[21] D.A. Parshin, H.R. Schober, V.L. Gurevich. Phys. Rev. B 76, 064206 (2007).

[22] W. Schirmacher, G. Diezemann, C. Ganter. Phys. Rev. Lett. 81, 136 (1998).

[23] M. Baggioli, A. Zaccone. Phys. Rev. Lett. 122, 145501 (2019).

[24] T. Nakayama. Rep. Prog. Phys. 65, 1195 (2002).

[25] H. Shintani, H. Tanaka. Nature Mater. 7, 870 (2008).

[26] S.N. Taraskin, Y.L. Loh, G. Natarajan, S.R. Elliott. Phys. Rev. Lett. 86, 1255 (2001).

[27] A.I. Chumakov, G. Monaco, A. Monaco, W.A. Crichton, A. Bosak, R. Ruffer, A. Meyer, F. Kargl, L. Comez, D. Fioretto, H. Giefers, S. Roitsch, G. Wortmann, M.H. Manghnani, A. Hushur, Q. Williams, J. Balogh, K. Parlinski, P. Jochym, P. Piekarz. Phys. Rev. Lett. 106, 225501 (2011).

[28] Л.Д. Ландау, Е.М. Лифшиц. Теория упругости. Наука, М. (1987). T. 7. 248 c.

[29] L. Wirtz, A. Rubio. Solid State Commun. 131, 141 (2004).

[30] J.C. Maxwell. Phil. Mag. 27, 294 (1865).

[31] Y.M. Beltukov, V.I. Kozub, D.A. Parshin. Phys. Rev. B 87, 134203 (2013).

[32] Y.M. Beltukov, D.A. Parshin. Письма в ЖЭТФ 104, 570 (2016).

[33] Д.А. Конюх, Я.М. Бельтюков, Д.А. Паршин. ФТТ 60, 369 (2018).

[34] Л.А. Фальковский. ЖЭТФ 142, 560 (2012).

[35] J.W. Kantelhardt, S. Russ, A. Bunde. Phys. Rev. B 63, 064302 (2001).
[36] T.S. Grigera, V. Martin-Mayor, G. Parisi. J. Phys.: Condens. Matter 14, 2167 (2002).

[37] K.S. Novoselov, A.K. Geim, S.V. Morozov, D. Jiang, M.I. Katsnelson, I.V. Grigorieva, S.V. Dubonos, A.A. Firsov. Nature 438, 197 (2005).

[38] P.R. Wallace. Phys. Rev. 71, 622 (1947).

[39] J.P. Hobson, W.A. Nierenberg. Phys. Rev. 89, 662 (1953)

[40] А. Марадудин, Э. Монтролл, Дж. Вейсс. Динамическая теория кристаллической решетки в гармоническом приближении. Мир, М. (1965). 383 с.

[41] Y.M. Beltukov, C. Fusco, D.A. Parshin, A. Tanguy. Phys. Rev. E 93, 023006 (2016).

[42] A. Weise, G. Wellein, A. Alvermann, H. Fehske. Rev. Mod. Phys. 78, 275 (2006).

[43] Y.M. Beltukov, D.A. Parshin. Письма в ЖЭТФ 93, 660 (2011).

[44] В.А. Марченко, Л.А. Пастур. Мат. сб. 72, 114, 507 (1967).

Редактор Ю.Э. Китаев 